\title{
Prevention of oral mucositis in cancer patients treated with chemotherapy or radiotherapy
}

\author{
What is the most effective intervention for the prevention of oral mucositis in \\ patients undergoing treatment for cancer?
}

\begin{abstract}
Stokman MA, Spijkervet FK, Boezen HM, Schouten JP, Roodenburg JL, de Vries EC. Preventive intervention possibilities in radiotherapy-and chemotherapy-induced oral mucositis: results of meta-analyses. J Dent Res 2006; 85:690-700
\end{abstract}

Data sources The Medline, Embase and CINAHL databases were used to source studies, along with the reference lists of identified articles.

Study selection Studies were restricted to randomised controlled trials (RCT), written in English, where the outcome of mucositis was recorded using the World Health Organization score or the NCI-CTC (National Cancer Institute-Common Toxicity Criteria) score, the absence or presence of ulcerations, or the presence or absence of grades 3 and 4 mucositis.

Data extraction and synthesis A total of 45 studies was included in a meta-analysis. When the included studies showed heterogeneity regarding the effect estimates, the results of the meta-analyses were based on the random-effects models; otherwise, the results were based on the fixed-effects models.

Results The search yielded 109 publications, 45 articles being included in the meta-analyses. These evaluated eight different interventions: local application of chlorhexidine; iseganan; PTA (polymyxin E, tobramycine and amphotericin B); granulocyte macrophage-colony-stimulating factor/ granulocyte colony-stimulating factor (GM-CSF/ G-CSF); oral cooling; sucralfate and glutamine; and systemic administration of amifostine and GM-CSF/G-CSF. Four interventions showed a significant preventive effect on the development or severity of oral mucositis: PTA [odds ratio (OR), 0.61; 95\% confidence interval (Cl), 0.39-0.96]; GM-CSF (OR, 0.53 ; $95 \% \mathrm{Cl}, 0.33-0.87)$; oral cooling (OR, 0.3; 95\% Cl, 0.16-0.56); and amifostine (OR, 0.37; 95\% Cl, 0.15-0.89).

Conclusions From current data, it can be concluded that no single intervention is capable of completely preventing oral mucositis. Future studies should evaluate a combination of interventions for the prevention of oral mucositis. In contrast, novel therapies could be developed that will improve outcomes and be used as single agents.

\section{Commentary}

Oral mucositis is a significant problem for patients undergoing treatment for cancer in general, and for head and neck cancers in particular. A systematic review by Trotti et al. ${ }^{1}$ noted that mucositis is a frequent and severe consequence of radiotherapy, occurring in the majority of patients being treated for head and neck cancer; the mean incidence is $80 \%$.

There have been a number of reviews of this area, of the traditional narrative type and also more systematic ones - most notably two Cochrane reviews looking at the prevention and treatment of oral mucositis in people receiving treatment for cancer. The recently updated Cochrane review on the prevention of oral mucositis is sum-

Address for correspondence: Dr MA Stokman, Department of Oral and Maxillofacial Surgery, University of Groningen and University Medical Centre Groningen, PO Box 30.001, 9700 RB Groningen, The Netherlands. E-mail: m.a.stokman@kchir.umcg.nl marised on page 104 of this issue of the journal.

The focus of this review by Stokman et al. is the prevention of oral mucositis in cancer patients treated with head and neck radiotherapy, chemotherapy or chemoradiation. The Medline, Embase and CINHAL databases and reference lists have been searched, but it is worth noting that the Cochrane Library was not utilised even though this is one of the best tools for finding systematic reviews and clinical trials. This and the fact that only English-language studies were included may have led to the exclusion of some relevant literature ${ }^{2}$. It is also surprising to note that the Cochrane reviews ${ }^{3-4}$ do not merit a mention, despite covering much of the same ground. Only RCT were included in the review, but no quality assessment of the trials was described and at least three of those included in this review were excluded on quality grounds by the Cochrane review. ${ }^{5-7}$

The review provides a very useful overview of a field that has expanded rapidly, with the development of more aggressive therapies for the treatment of cancer and improved survival rates and consequent cases of oral mucositis. The figure in this review that outlines possible pathways of interventions for mucositis prevention is very useful: it indicates where the 27 different interventions identified here for the prevention of oral mucositis may act.

Both this review and the Cochrane review find that some of the interventions have some benefit, and agree on three: amifostine, antibiotic paste or pastille, and oral cooling (ice chips). There is also agreement that there is a need for well-designed and -conducted trials with sufficient numbers of participants. I find it difficult to agree with the authors' conclusion, however, that because no single intervention completely prevents oral mucositis, combined preventive therapy strategies seem to be required to ensure more successful outcomes. Before we throw the kitchen sink at the problem we should first conduct appropriately powered high quality studies with single interventions

\section{Derek Richards}

Director, Centre for Evidence-based Dentistry, Oxford, UK

1. Trotti A, Bellm LA, Epstein JB, et al. Mucositis incidence, severity and associated outcomes in patients with head and neck cancer receiving radiotherapy with or without chemotherapy: a systematic literature review. Radiother Oncol 2003; 66:253-262.

2. Juni P, Holenstein F, Sterne J, Bartlett C, Egger M. Direction and impact of language bias in meta-analyses of controlled trials: empirical study. Int J Epidemiol 2002; 31:115-123.

3. Clarkson JE, Worthington HV, Eden OB. Interventions for preventing oral mucositis for patients with cancer receiving treatment (Cochrane Review). Cochrane Library 2003; issue 3. Oxford: Update Software.

4. Worthington HV, Clarkson JE, Eden OB. Interventions for treating oral mucositis for patients with cancer receiving treatment. Cochrane Database Syst Rev 2004; issue 2:

5. Cheng KK, Chang AM, Yuen MP. Prevention of oral mucositis in paediatric patients treated with chemotherapy; a randomised crossover trial comparing two protocols of oral care. Eur J Cancer 2004 40:1208-1216.

6. Okuno SH, Foote RL, Loprinzi CL, et al. A randomized trial of a nonabsorbable antibiotic lozenge given to alleviate radiation-induced mucositis. Cancer 1997; 79:2193-2199.

7. El-Sayed S, Epstein J, Minish E, Burns P, Hay J, Laukkanen E. A pilot study evaluating the safety and microbiologic efficacy of an economically viable antimicrobial lozenge in patients with head and neck cancer receiving radiation therapy. Head Neck 2002; 24:6-15.

Evidence-Based Dentistry (2006) 7, 81-82. doi:10.1038/sj.ebd.6400452 